\title{
Comparison of Chiral Fermion Methods
}

\author{
Robert G. Edwards*† \\ Jefferson Lab, 12000 Jefferson Ave, Newport News, VA 23606, USA. E-mail: \\ edwards@jlab.org
}

\section{Bálint Joó}

UKQCD Collaboration

School of Physics, University of Edinburgh, King's Buildings

Edinburgh EH9 3JZ, UK

E-mail: bjoo@jlab.org

\section{Anthony D. Kennedy}

School of Physics, University of Edinburgh, King's Buildings

Edinburgh EH9 3JZ, UK

E-mail: adk@ph.ed.ac.uk

\section{Kostas Orginos}

Jefferson Lab and Department of Physics, College of William and Mary

P.O. Box 8795, Williamsburg, VA 23187-8795, USA

E-mail: kostasejlab.org

\section{Urs Wenger ${ }^{\S}$}

John von Neumann Institut für Computing NIC

Platanenallee 6, D-15738 Zeuthen, Germany

E-mail: urs.wengerdesy.de

We present a comparison of various five-dimensional representations of chiral fermions considering their cost and residual chiral symmetry breaking.

XXIIIrd International Symposium on Lattice Field Theory

25-30 July 2005

Trinity College, Dublin, Ireland

\footnotetext{
* Speaker.

${ }^{\dagger}$ Submitted to PoS on 12th October 2005.

¥Address since September 16, 2005: Jefferson Lab, 12000 Jefferson Ave, Newport News, VA 23606, USA

$\S$ Address since 1st October 2005: Institute for Theoretical Physics, ETH Zürich, CH-8093 Zürich, Switzerland.
} 


\section{Introduction}

The realization of chiral symmetry on the lattice represents a major breakthrough in the field of nonperturbative studies of QCD - in particular it allows the numerical simulation of QCD on the lattice with realistically light fermion flavors. The key to this success is the overlap Dirac operator which is related to the domain wall formalism in five dimensions [1, 2, 国, 代.

This paper provides a comprehensive discussion of various 5D versions of the overlap Dirac operator where the matrix sign function is approximated by a rational function. We show explicitly how the different variants emerge from the different representations of the rational function and how they all reduce to the same $4 \mathrm{D}$ effective lattice Dirac operator which is the usual overlap operator or its Hermitian version. In particular we show that the standard domain wall formulation is simply just one specific member of a large class of 5D operators.

An important goal of the numerical work is to evaluate the "cost" of various chiral fermion actions. We only consider 5D operators - the ultimate aim is their use in the force term in Hybrid Monte Carlo simulations.

We will consider 5D formulations to the 4D Overlap Operator or its Hermitian form:

$$
\begin{aligned}
D_{4}(m) & =\frac{1}{2}\left[(1+m)+(1-m) \gamma_{5} \operatorname{sgn}(H)\right] \\
D_{4}^{H}(m) & =\frac{2}{1-m} \gamma_{5} D_{4}(m)=R \gamma_{5}+\operatorname{sgn}(H), \quad R=\frac{1+m}{1-m}
\end{aligned}
$$

where $\operatorname{sgn}(H)$ is approximated by a rational function $\varepsilon(H)$. There is a four dimensional space of algorithms we can exploit:

- choice of kernel $H$,

- representation (form) of $\varepsilon(H)$. We consider partial fraction, continued fraction and Euclidean Cayley Transform representations,

- approximation of $\operatorname{sgn}(H) \approx \varepsilon_{n, m}(H)=\frac{P_{n}(H)}{Q_{m}(H)}$ for some polynomials $P_{n}$ and $Q_{m}$. This is usually the set of coefficients that fix the particular approximation in the chosen representation, and

- constraints - how are fermions introduced into the path integral.

Let us consider the various representations of a rational function. The Partial Fraction form [5, 6, 7] uses

$$
\varepsilon_{2 n-1,2 n}(x)=x\left(p_{0}+\sum_{i=1}^{n} \frac{p_{i}}{x^{2}+q_{i}}\right)
$$

The Continued Fraction form [8, 9, 10] uses

$$
\varepsilon_{2 n-1,2 n}(x)=\beta_{0} x+\frac{1}{\beta_{1} x+\frac{1}{\ldots+\frac{1}{\beta_{2 n} x}}}
$$




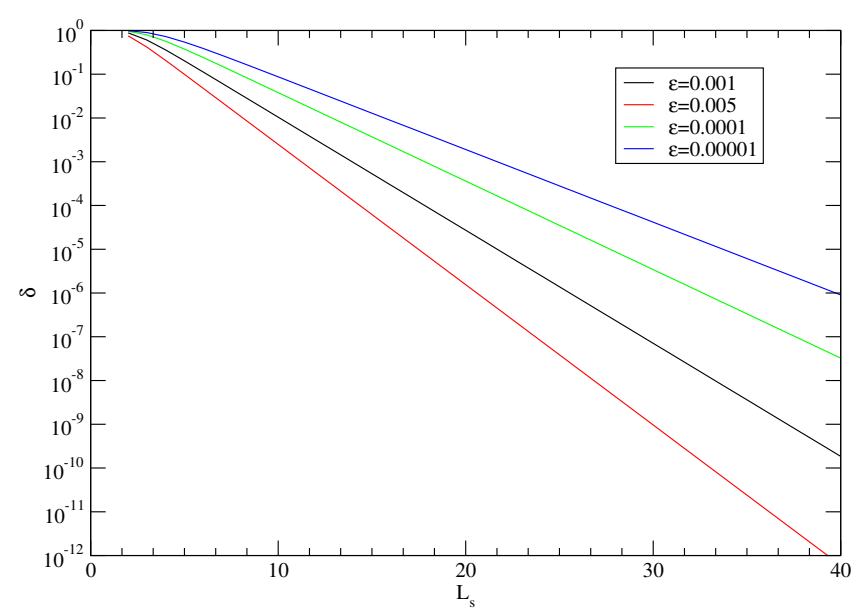

Figure 1: Maximal error of the Zolotarev approximation over the region $[\varepsilon, 1]$ as a function of $\varepsilon$ and $L_{s}$.

The Euclidean Cayley Transform form connects the overlap form to the domain wall form [7 11 . 12, 13, 14. It uses

$$
\varepsilon_{2 n-1,2 n}(x)=\frac{T^{-1}(x)-1}{T^{-1}(x)+1}, \quad T(x)=\prod_{i=1}^{2 n} \frac{1-\omega_{i} x}{1+\omega_{i} x}
$$

$T$ is the transfer matrix in the fifth dimension, and the $\omega_{i}$ come from the specific approximation.

There are various approximation functions for $\operatorname{sgn}(H)$. These approximations give coefficients for all the representations mentioned above. One can also supplement the approximation with eigenvectors of the $H$ operator to decrease errors [6]; projection can be used in all representations [12]. The original Higham-Tanh approximation [15] (a.k.a. Neuberger's Polar form [5]) is

$$
\varepsilon_{2 n-1,2 n}(x)=\tanh \left(-2 n \ln \left|\frac{1-x}{1+x}\right|\right)=\tanh \left(2 n \tanh ^{-1}(x)\right)=\frac{(1+x)^{2 n}-(1-x)^{2 n}}{(1+x)^{2 n}+(1-x)^{2 n}} .
$$

We note that $\varepsilon_{2 n-1,2 n}(x)=\varepsilon_{2 n-1,2 n}(1 / x)$.

Over a desired approximation region $(x \in[\varepsilon, 1])$, the approximation due to Zolotarev

$$
\varepsilon_{2 n, 2 n-1}(x)=x R_{n, n}\left(x^{2}\right), \quad R_{n, n}\left(x^{2}\right)=A \frac{\prod_{l=1}^{n}\left(x^{2}+\bar{c}_{2 l}\right)}{\prod_{l=1}^{n}\left(x^{2}+\bar{c}_{2 l-1}\right)}
$$

with

$$
\bar{c}_{2 l}=-\kappa^{2} \mathrm{sn}^{2}(2 i K l / n, \kappa) \quad \bar{c}_{2 l-1}=-\kappa^{2} \mathrm{sn}^{2}\left(i K \frac{2 l-1}{n}, \kappa\right)
$$

has much reduced errors for a fixed number of poles compared to the "tanh" approximation. Here, $\kappa=\sqrt{1-\varepsilon^{2}}$ and $K=K(\kappa)$ is the complete elliptic integral. The $\omega_{j}$ are the locations in $x$ where $\varepsilon_{2 n-1,2 n}(x)=1$.

We plot in Figure 1 the maximal error of the Zolotarev approximation over the interval $[\varepsilon, 1]$ as a function of $L_{s}$ for various values of $\varepsilon$. The maximal error of the tanh approximation over the same interval is very close to 1 - the maximal error occurs at $x=\varepsilon$. 
The kernel $H$ - the auxiliary Dirac operator - is what determines the "physics" of the problem. For example, this is where the discretization errors enter. There are an infinite number of choices here, but some common variants are as follows. The overlap-like version is $H=H_{w}=\gamma_{5} D_{w}(-M)$ where $D_{w}(-M)$ is the Wilson-Dirac operator with supercritical mass ${ }^{1} 0<M<2$. In the Shamir domain wall version, the induced operator is $H=H_{T}=H_{w}\left(2+a_{5} D_{w}\right)^{-1}$. The Möbius version [14] $H=\left(b_{5}+c_{5}\right) H_{w}\left[2+\left(b_{5}-c_{5}\right) D_{w}\right]^{-1}$ interpolates between $H_{w}$ and $H_{T}$.

One could use smeared links within the operator to improve the spectrum (see e.g. [16]). We require $H$ to be local, and differentiable (for dynamical fermion calculations). Ideally, the approximation should cover the spectrum of $H$. Different choices of $H$ lead to different spectra and different $O(a)$-discretization effects in $H$ and therefore to different $O\left(a^{2}\right)$ effects in the resulting $D_{4}$ operator.

\section{Introducing the Schur Decomposition}

The framework we shall use for 5D chiral fermion operators is based on Schur decomposition. Using a 5D form gives the obvious benefit of inversion of 4D chiral operator within a single (5D) Krylov space. Furthermore, this framework allows for a formal reduction of the 5D theory to the 4D theory. It also allows for a formal derivation of the determinant of the 5D operator for 5D dynamical fermion calculations. Even-odd preconditioning can also be accommodated in this framework.

Consider the 5D block matrix

$$
M_{5}=\left(\begin{array}{ll}
A & B \\
C & D
\end{array}\right)
$$

with $D$ a 4 D sub-matrix. The Schur decomposition of $M_{5}$ is:

$$
M_{5}=\tilde{L} \tilde{D} \tilde{U}=\underbrace{\left(\begin{array}{cc}
1 & 0 \\
C A^{-1} & 1
\end{array}\right)}_{\tilde{L}} \underbrace{\left(\begin{array}{ll}
A & 0 \\
0 & S
\end{array}\right)}_{\tilde{D}} \underbrace{\left(\begin{array}{cc}
1 & A^{-1} B \\
0 & 1
\end{array}\right)}_{\tilde{U}} \text { where } S=D-C A^{-1} B
$$

is referred to as the Schur Complement. The essence of the 5D framework is that $D_{4}(m)$ (or $D_{4}^{H}(m)$ ) is the 4D Schur Complement of $M_{5}$ (or of a unitary transformation of $M_{5}$ ). The use of the Schur Complement in 5D formulations was advocated in 7 in connection with multigrid techniques.

The Schur decomposition automatically provides inversion of the 4D system through inversion of the 5D system. We have

$$
M_{5}\left(\begin{array}{c}
\phi \\
\psi_{4}
\end{array}\right)=\left(\begin{array}{c}
0 \\
\chi_{4}
\end{array}\right) \Rightarrow \overbrace{\left(\begin{array}{cc}
A & 0 \\
0 & S
\end{array}\right)}^{\tilde{D}} \overbrace{\left(\begin{array}{c}
\phi+A^{-1} B \psi_{4} \\
\psi_{4}
\end{array}\right)}^{\tilde{U}\left(\begin{array}{c}
\phi \\
\psi_{4}
\end{array}\right)}=\overbrace{\left(\begin{array}{c}
0 \\
\chi_{4}
\end{array}\right)}^{\tilde{L}^{-1}\left(\begin{array}{c}
0 \\
\chi_{4}
\end{array}\right)} .
$$

and it is clear, that solving the $5 \mathrm{D}$ system solves the $4 \mathrm{D}$ system $S \psi_{4}=\chi_{4}$.

\footnotetext{
${ }^{1}$ Also referred to as the domain wall height
} 
The Schur decomposition also provides the determinant and the effective 4D operator. By inspection of eq. (2.2), we see that $\operatorname{det}(\tilde{L})=\operatorname{det}(\tilde{U})=1 \Rightarrow \operatorname{det}\left(M_{5}\right)=\operatorname{det}(A) \operatorname{det}(S)$. As a consequence, we need an operator that will cancel bulk 5D modes in $M_{5}$ (i.e., $\operatorname{det}(A)$ ). This operator is often called the Pauli-Villars operator which we define here as follows:

$$
M_{P V}=\tilde{L} \tilde{D}_{P V} \tilde{U}, \quad \tilde{D}_{P V}=\left(\begin{array}{cc}
A & 0 \\
0 & 1
\end{array}\right) \Rightarrow \operatorname{det}\left(M_{P V}\right)=\operatorname{det}\left(\tilde{D}_{P V}\right)=\operatorname{det}(A)
$$

Formally at least, $M_{5} M_{P V}^{-1}$ is an effective $4 \mathrm{D}$ operator:

$$
M_{5} M_{P V}^{-1}\left(\begin{array}{c}
0 \\
\psi_{4}
\end{array}\right)=\tilde{L}\left(\begin{array}{ll}
1 & 0 \\
0 & S
\end{array}\right) \tilde{L}^{-1}\left(\begin{array}{c}
0 \\
\psi_{4}
\end{array}\right)=\left(\begin{array}{c}
0 \\
S \psi_{4}
\end{array}\right) .
$$

We can now connect the Schur complement formalism to the 5D action and path integral (the constraints). Requiring the existence of $A^{-1}$ and the positivity of $S$ only, we have for the partition function:

$$
\begin{aligned}
Z & =\int d U d \psi d \bar{\psi} \exp \left\{-\bar{\psi}\left(M_{5} M_{P V}^{-1}\right) \psi\right\}=\int d U d \psi d \bar{\psi} \exp \left\{-\bar{\psi} \tilde{L}\left(\begin{array}{ll}
1 & 0 \\
0 & S
\end{array}\right) L^{-1} \psi\right\} \\
& =\int d U d \chi d \bar{\chi} \exp \left\{-\bar{\chi}\left(\begin{array}{ll}
1 & 0 \\
0 & S
\end{array}\right) \chi\right\}, \quad \text { defining } \bar{\chi}=\bar{\psi} \tilde{L}, \chi=\tilde{L}^{-1} \psi \\
& \propto \int d U d \chi_{4} d \bar{\chi}_{4} \exp \left\{-\bar{\chi}_{4} S \chi_{4}\right\} .
\end{aligned}
$$

The path integral needs $M_{P V} M_{5}^{-1}$, but in general $M_{P V}$ involves $A^{-1}$ (in $\tilde{L}$ ) which can be a dense operator. However, one can always construct $\tilde{D}_{P V}$ which needs only $A$. The resulting partition function is

$Z=\int d U d \phi^{\dagger} d \phi d \eta^{\dagger} d \eta \exp \left\{-\phi^{\dagger} M_{5}^{-1} \phi-\eta^{\dagger} \tilde{D}_{P V} \eta\right\}=\int d U \operatorname{det}(A) \operatorname{det}(S) / \operatorname{det}(A)=\int d U \operatorname{det}(S)$.

In Hybrid Monte Carlo, one uses $M_{5}^{\dagger} M_{5}, \tilde{D}_{P V}^{\dagger} \tilde{D}_{P V}$ to ensure integrals converge. Single flavor theories can be simulated with RHMC and taking the square and fourth roots of the squared operators.

\section{Matrix Representations}

We now turn to the specific 5D representations used in this work. For purposes of illustration, we will consider unpreconditioned systems with short 5D extents.

Partial Fraction: Let us begin with the partial fraction operator of ref. [17]. We show below partial fraction expansion with only two poles. The operator is

$$
M_{5}=\left(\begin{array}{cccc|c}
H & -\sqrt{q_{2}} & 0 & 0 & 0 \\
-\sqrt{q_{2}} & -H & 0 & 0 & \sqrt{p_{2}} \\
0 & 0 & H & -\sqrt{q_{1}} & 0 \\
0 & 0 & -\sqrt{q_{1}} & -H & \sqrt{p_{1}} \\
\hline 0 & \sqrt{p_{2}} & 0 & \sqrt{p_{1}} & R \gamma_{5}+p_{0} H
\end{array}\right)=\left(\begin{array}{c|c|c}
A & B \\
\hline C & D
\end{array}\right),
$$


and the Schur Complement is just the pole approximation to $D_{4}^{H}(m)$ :

$$
S=R \gamma_{5}+p_{0} H+\frac{p_{1}}{H+q_{1} H^{-1}}+\frac{p_{2}}{H+q_{2} H^{-1}}=R \gamma_{5}+H\left(p_{0}+\sum_{i=1}^{2} \frac{p_{i}}{H^{2}+q_{i}}\right) \approx D_{4}^{H}(m)
$$

and all the results of the section 2 follow.

Continued Fraction: For the continued fraction representation, we illustrate the matrix in the $2 n+1=3$ case:

$$
M_{5}=\left(\begin{array}{ccc}
\beta_{2} H & 1 & 0 \\
1 & -\beta_{1} H & 1 \\
0 & 1 & R \gamma_{5}+\beta_{0} H
\end{array}\right)
$$

We apply the Schur decomposition recursively. In order to form $S$ we need to invert it using its Schur complement $S_{1}$ and so forth. Finally we arrive at

$$
M_{5}=\left(\begin{array}{ccc}
1 & 0 & 0 \\
S_{2}^{-1} & 1 & 0 \\
0 & S_{1}^{-1} & 1
\end{array}\right)\left(\begin{array}{ccc}
S_{2} & 0 & 0 \\
0 & S_{1} & 0 \\
0 & 0 & S
\end{array}\right)\left(\begin{array}{ccc}
1 & S_{2}^{-1} & 0 \\
0 & 1 & S_{1}^{-1} \\
0 & 0 & 1
\end{array}\right)
$$

where

$$
\begin{aligned}
S_{2} & =\beta_{2} H, \quad S_{1}=-\beta_{1} H-S_{2}^{-1} \\
S & =R \gamma_{5}+\beta_{0} H-S_{1}^{-1}=R \gamma_{5}+\beta_{0} H+\left(\beta_{1} H+\left(\beta_{2} H\right)^{-1}\right)^{-1} \\
& \approx D_{4}^{H}(m)
\end{aligned}
$$

and the outermost Schur complement is $S=D_{4}^{H}$ and all the previous results apply.

Domain Wall: The domain wall formalism can be written in a Cayley transform Schur complement system [17, 11, 12, 13, 14]. To simplify the formalism, we apply a unitary transformation (defined in [12]) on the domain wall kernel to bring each chiral half of the physical field onto the same wall. We find that (using a length of $2 n=4$ in the 5 th dimension for illustration):

$$
Q_{-}^{-1} \gamma_{5} M_{5} \mathscr{P}=\left(\begin{array}{ccc|c}
1 & -T_{2}^{-1} & 0 & 0 \\
0 & 1 & -T_{3}^{-1} & 0 \\
0 & 0 & 1 & -T_{4}^{-1} c_{+} \\
\hline-T_{1}^{-1} & 0 & 0 & c_{-}
\end{array}\right),
$$

where

$$
T_{i}=\frac{1-\omega_{i} H}{1+\omega_{i} H}, \quad c_{ \pm}=\frac{1-m}{2} \pm \frac{1+m}{2} \gamma_{5}
$$

The Schur complement is:

$$
\begin{aligned}
S & =c_{-}+T_{1}^{-1} T_{2}^{-1} T_{3}^{-1} T_{4}^{-1} c_{+}, \quad \text { define } T^{-1}=T_{1}^{-1} T_{2}^{-1} T_{3}^{-1} T_{4}^{-1} \\
& =-\left(T^{-1}+1\right) \gamma_{5}\left[\frac{1+m}{2}+\frac{1-m}{2} \gamma_{5} \frac{T^{-1}-1}{T^{-1}+1}\right]=M_{5}(m=1) D_{4}(m)
\end{aligned}
$$

We find that the domain wall system is a little different from the others. We see that $\operatorname{det}(A)=1$ and $S=M_{5}(m=1) D_{4}(m)$. On the other hand, one can define $M_{P V}=M_{5}(m=1)$ which simplifies its use and provides a local 5D operator. This result implies $M_{P V}^{-1} M_{5}=D_{4}(m)$ is an effective 4D operator. Remaining results then follow from the Schur complement machinery. 


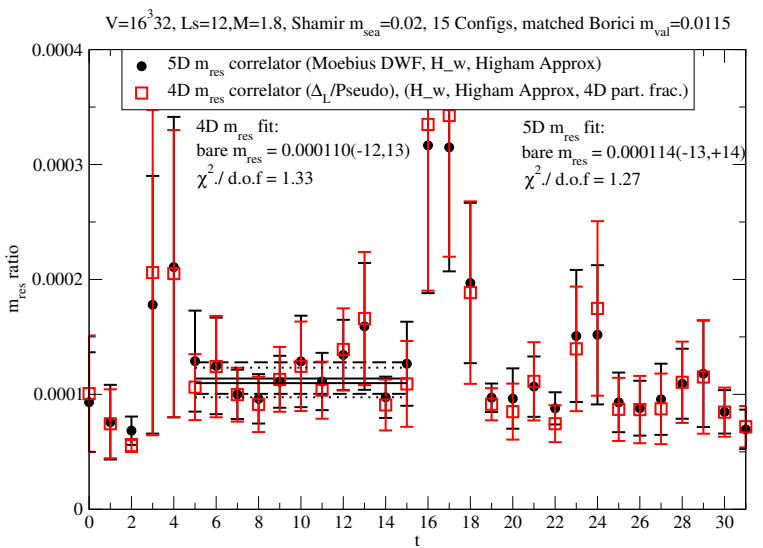

Figure 2: Comparison of the determination of $m_{\text {res }}$ using either a fit in $4 \mathrm{D}$ or $5 \mathrm{D}$ to $m_{\text {res }}(t)$.

Preconditioning and Implementation Tricks: Suppose our operator $H$ is a ratio of two operators, $H=H_{N} H_{D}^{-1}$ (e.g., the kernel is $H_{T}$ or $H_{\text {Moebius. }}$ ) In this case, we naively have an inversion in our $5 \mathrm{D}$ operator, resulting in a nested inversion. We overcome this, by rationalizing the denominator and solving $M_{5} H_{D} H_{D}^{-1} \psi=\chi$. We proceed in two steps, first solving $\left(M_{5} H_{D}\right) \psi^{\prime}=\chi$, then $\psi=H_{D} \psi^{\prime}$. After collecting terms in $M_{5} H_{D}$, we have just one extra $H$ application to reconstruct $\psi$.

All three formulations allow continuous equivalence transformations on the approximation coefficients which may be used to make the operator better conditioned.

Even-odd preconditioning follows directly from the Schur decomposition framework developed so far. The main consideration is the even-odd block decomposition of the 5D operator ${ }^{2}$. If

$$
M_{5}=\left(\begin{array}{ll}
M_{e e} & M_{e o} \\
M_{o e} & M_{o o}
\end{array}\right)=\left(\begin{array}{cc}
1 & 0 \\
M_{o e} M_{e e}^{-1} & 1
\end{array}\right)\left(\begin{array}{cc}
M_{e e} & 0 \\
0 & M_{o o}-M_{o e} M_{e e}^{-1} M_{e o}
\end{array}\right)\left(\begin{array}{cc}
1 & M_{e e}^{-1} M_{e o} \\
0 & 1
\end{array}\right)
$$

and if $M_{e e}$ preserves the structure of $M_{5}$, then the Schur decomposition of $M_{5}$ suggests the efficient application of $M_{e e}^{-1}$ : one should Schur decompose $M_{e e}$.

For the representations presented here, the efficient use of even-odd preconditioning depends on $H$. For general $H=H_{\text {Moebius, }}$ we see that $M_{e e}^{-1}$ does not depend on the gauge fields and can be efficiently applied to a vector.

\section{Numerical Results}

\subsection{Which 5D Fermion Action?}

We want to evaluate the "cost" of various chiral fermion actions. Here, we only consider 5D inversions for use in the force term in HMC and no eigenvector projection is used. We thus have a residual mass. We choose a winner using a cost metric, which is the number of Wilson-Dirac applications for fixed residual mass. Using the denominator rationalization trick mentioned earlier we find that if a general Möbius DWF operator requires $2 n$ applications of $D_{w}$, the corresponding

\footnotetext{
${ }^{2}$ This is also a Schur Decomposition
} 

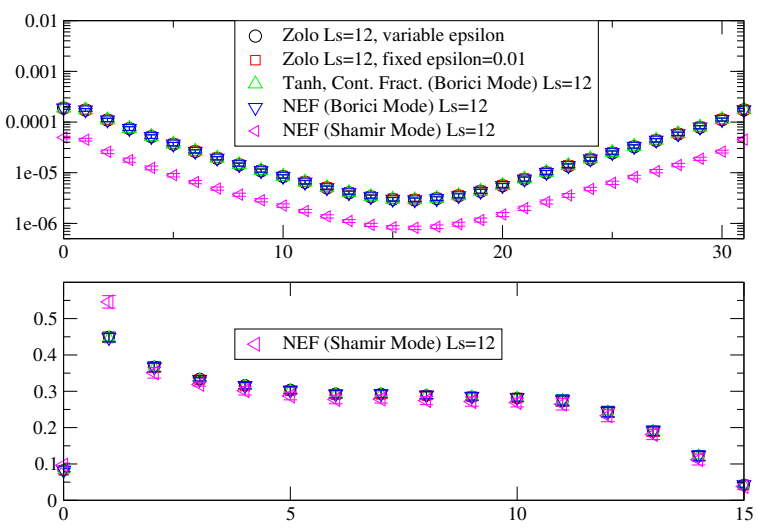

Figure 3: Comparison of the pion correlator and effective mass for various actions, demonstrating how different variants of 5D operators reduce to the same effective 4D operator. The different kernels $H_{w}$ and $H_{T}$ produce different pion correlators, but the pion mass is tuned to the same value. The difference in mass coming from the difference between the tanh and Zolotarev coefficients is too small to show up on these plots.
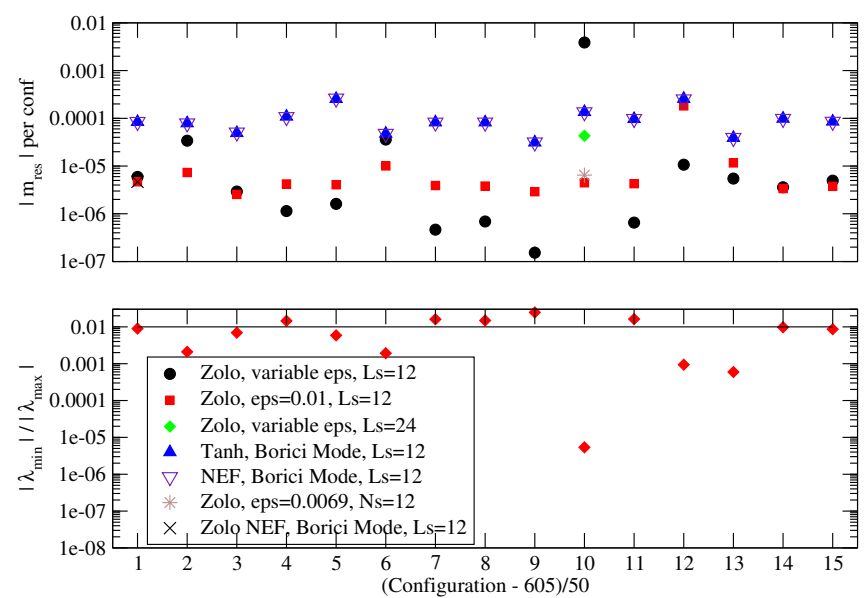

Figure 4: In the upper panel is shown the residual mass per configuration for various 5D actions using $H=$ $H_{w}$. Here, "Zolo" is the Zolotarev version of Continued Fraction, "Tanh" is the tanh version of Continued Fraction, "NEF" is the tanh domain-wall like variant, and "Zolo NEF" is the Zolotarev domain-wall like variant. In the lower panel is the ratio of extremal eigenvalues for $H_{w}$ per configuration.

Continued or Partial fraction operators require $2 n+1$ such applications. In the case of $H=H_{w}$, all formulations require only $2 n$ applications of $D_{w}$ (since $\beta_{0}=0$ in the continued fraction and $p_{0}=0$ in the partial fraction case).

\subsection{Residual chiral symmetry breaking}

For our cost comparisons, we note that the 5D definition of the residual mass has been related 
to the $4 \mathrm{D}$ effective operator [14, 18]:

$$
m_{\mathrm{res}}=\frac{\sum_{x}\left\langle\bar{Q}_{x} \gamma_{5} Q_{x} \bar{q}_{0} \gamma_{5} q_{0}\right\rangle}{(1-m)^{2}\left\langle\sum_{x} \bar{q}_{x} \gamma_{5} q_{x} \bar{q}_{0} \gamma_{5} q_{0}\right\rangle}=\frac{\sum_{x} \operatorname{Tr}\left(D_{4}^{\dagger}(m)\right)_{x, 0}^{-1} \Delta_{x, y} D_{4}^{-1}(m)_{y, 0}}{\sum_{x} \operatorname{Tr} G_{x, 0}^{\dagger} G_{x, 0}}
$$

Here, $G=\frac{1}{1-m}\left(D_{4}^{-1}(m)-1\right)$ is the usual subtracted propagator of the $4 \mathrm{D}$ overlap formalism, and $\Delta$ is the breaking term in the Ginsparg-Wilson relation from the approximation where $\varepsilon \neq \operatorname{sgn}$ :

$$
2 \gamma_{5} \Delta=\gamma_{5} D_{4}(0)+D_{4}(0) \gamma_{5}-2 D_{4}(0) \gamma_{5} D_{4}(0) \Rightarrow \Delta=\frac{1}{4}\left(1-\varepsilon^{2}(H)\right)
$$

where we have used $D_{4}(0)=\frac{1}{2}\left(1+\gamma_{5} \varepsilon(H)\right)$. We see that $m_{\text {res }} \neq 0$ is purely a deficiency of the approximation $\varepsilon(H) \approx \operatorname{sgn}(H)$. Further, the numerator in eq. (4.1) can easily be computed in $4 \mathrm{D}$ using the pole approximation of $\varepsilon(H)$. Thus, we can compare chiral breaking for all formulations on an equal footing.

Summation is carried out over both spatial and temporal coordinates in 4.1. If one does not sum over time, the resulting time-sliced correlation function is not identically equal to the usual Domain Wall $m_{\text {res }}(t)$. However, since $\Delta_{x, y}$ is a local operator, at low energies the two are very similar as can be seen by observing fig. 2. Further, at the cost of introducing a small contact term on the source time-slice and a term that vanishes in the ensemble average one may replace the $D^{-1}$ terms in eq. (4.1) by the subtracted propagators $G$. In our computations, this simplification was used, however, apart from figure 2 we always carried out the temporal sum.

\subsection{Setup}

For our numerical tests, we used $15 N_{f}=2$ domain-wall fermion configurations (provided by RBC [19]) with $L_{s}=12$ and $a m_{q}=0.02$ corresponding to $m_{\pi}=500 \mathrm{MeV}$. We compare the cost of inversions for even-odd preconditioned Möbius variants of 5D Partial Fraction, Continued Fraction, and Domain Wall operators. We use only a CGNE inverter. Unfortunately, BiCG-stab and MR are not convergent. In all tests, we used a target residual of $10^{-7}$ and $M=1.8$ in $D_{w}$. We tuned the pion mass of our 5D operators using $H=H_{w}$ to match the domain-wall pion mass which resulted in an $a m_{q}=0.0115$.

We computed the cost (number of Wilson-Dirac applications) for multiple $L_{s}$ with the tanh and Zolotarev approximations. For the latter, we found the largest eigenvalue of $H_{T}$ and $H_{w}$ to be about 1.6 and 5.8, respectively. We set the upper bound of the approximation to this $\lambda_{\max }$, and set the lower bound to a fixed $\varepsilon \times \lambda_{\max }$ where $\varepsilon=0.01$ in practice.

\subsection{Equivalence of representations/variants}

In Figure 3, we show the pion correlator for different representations and approximations at the tuned pion mass. We see that the deviations from each other are small and under control.

\section{$4.5 m_{\text {res }}$ per configuration}

In Figure 7 we show a plot of $m_{\text {res }}$ per configuration for various actions using $H=H_{w}$. We see that the "tanh" versions of domain-wall and continued fraction agree as expected. Also, the Zolotarev versions of domain-wall and continued fraction agree for the first configuration. We did 


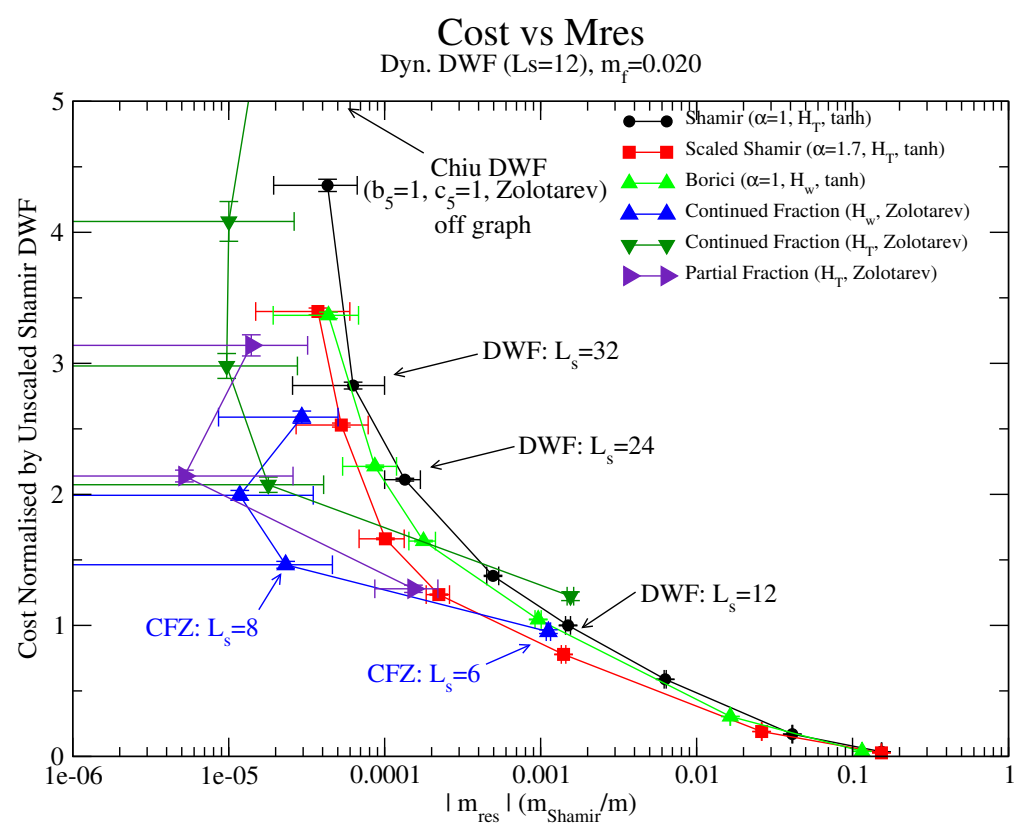

Figure 5: Cost in Wilson-Dirac applications vs. $m_{\text {res }}$ for the most promising 5D actions.

not measure the Zolotarev version of the domain wall for the remaining configurations as the cost appeared too expensive, however, we expect similar agreement we see for the first configuration. We also see that in the "tanh" approximation cases the $m_{\text {res }}$ is notably higher than in the Zolotarev cases. This is consistent with the much larger approximation errors $\varepsilon(x)$ in polar form compared to Zolotarev.

The notable exception to a small $m_{\text {res }}$ is configuration 10 where the lowest eigenvalue of $H_{w}$ is quite small. We find that fixing the approximation range is preferable to allowing it to vary. What we are seeing is an interplay of the density of eigenvalues of $H_{w}$ and the approximation accuracy. The eigenvectors of $H_{w}$ are also eigenvectors of $\Delta$ which can be written as $\int d \lambda \rho(\lambda) \Delta_{L}(\lambda)=$ $(1 / 4) \int d \lambda \rho(\lambda)\left(1-\varepsilon(\lambda)^{2}\right)$. If the density of small modes is low, it is not worth increasing the approximation range to accommodate the low modes. Increasing the approximation range increases the maximum error which thus magnifies $\Delta$ for all modes - not just the smallest modes. In our tests we therefore fixed the maximal range of the approximation. We note that is no worse than a "tanh" approximation where the approximation is fixed for a given order of the rational approximation.

\subsection{Cost versus $m_{\text {res }}$}

In our tests we compare the cost of inverting the even-odd preconditioned 5D operators versus $m_{\text {res }}$. We note that this preconditioning gives between a factor of 1.5-3 speedup over the unpreconditioned systems. The cost of applying $M_{e e}^{-1}$ is negligble in flops; however, some care is needed in writing optimized codes due to memory loads in $M_{e e}$. The goal is small $m_{\mathrm{res}}$ for small cost.

In Figure 5 we plot the cost versus $m_{\text {res }}$ for the most promising actions. Many of the Zolotarev variants were very expensive and not shown. We remark, however, that we did not explore possible further tuning of the condition of our operators via equivalence transformations on our coefficients, which may reduce the cost of these expensive variants. Having said this, of all the actions we 


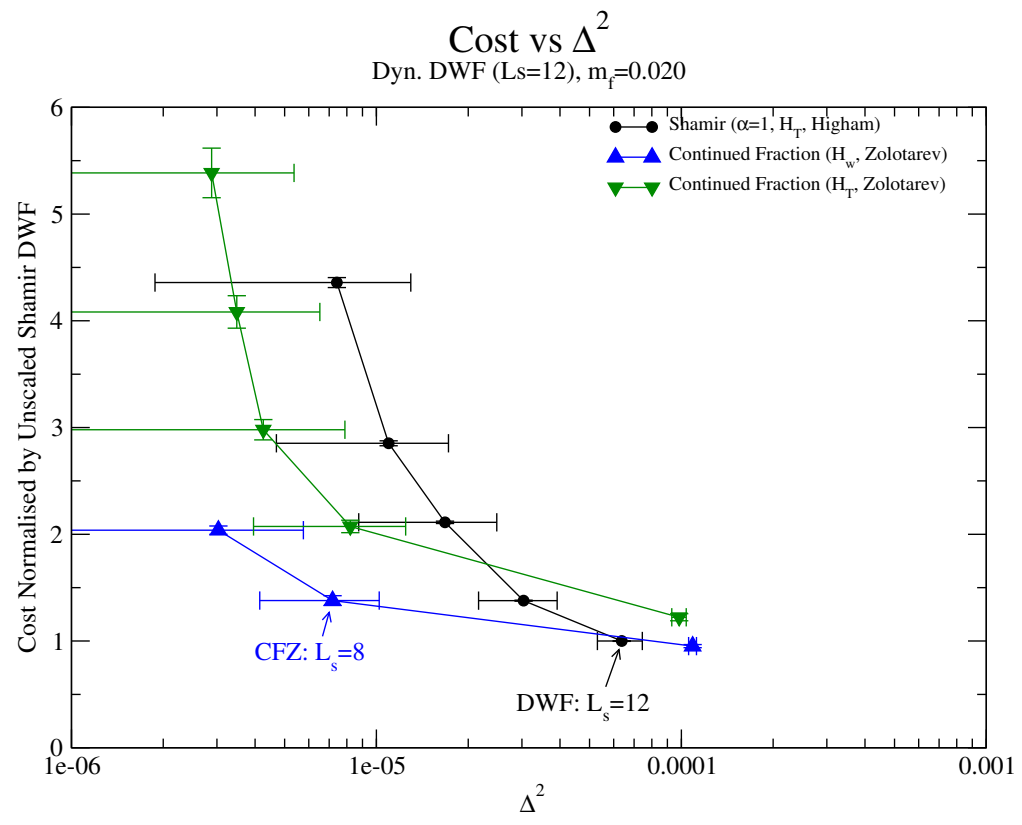

Figure 6: Cost in Wilson-Dirac applications vs. $\Delta^{2}$ for some of the $5 \mathrm{D}$ actions in Figure

tested, the standard domain-wall $\left(H_{T}\right)$ is the least effective. The Zolotarev Continued Fraction $\left(H_{w}\right.$ and $\left.H_{T}\right)$ are the top candidates. The Zolotarev Partial Fraction version is promising. We note that rescaling the coefficients $b_{5}+c_{5}$ in $H_{\text {Moebius }}$ with $b_{5}-c_{5}=1$ is equivalent to a rescaling $\alpha=b_{5}+c_{5}$ in $\varepsilon\left(\alpha H_{T}\right)$. For the tanh approximation, we can slide the $H_{T}$ down inside $\varepsilon\left(H_{T}\right)$ to lower approximation errors. We see this rescaling results in a corresponding decrease in $m_{\text {res }}$ at no cost increase.

In Figure 6 we plot the cost versus the second moment $\Delta^{2}$. We see no large shift in cost corresponding to wild oscillations that cancel in $m_{\text {res }}$ but not in $\Delta^{2}$.

\section{Summary and conclusions}

We have presented a unified framework for 5D chiral operators. The crux of the framework is the Schur decomposition and Schur complement techniques. The framework is agnostic about representation, approximation or the kernel. The framework accommodates (and can help with) even-odd preconditioning. In practice, the computational cost of a 5D formulation depends upon the spectral properties of $M_{5}$ and upon $m_{\text {res }}$. While $m_{\text {res }}$ is an artifact of the approximation $\varepsilon(H)$, it depends not only on how good the approximation is, but also how much of the spectrum of $H$ is not well approximated (e.g., the near-zero modes of $H$ ). The condition of $M_{5}$ depends upon its matrix structure and coefficients, e.g., the approximation and representation and potentially on further tuning of the condition through equivalence transformations and permutation symmetry.

We have presented a detailed comparison of the "cost" of various chiral fermion actions, where cost is the number of Wilson-Dirac applications for fixed $m_{\text {res }}$ in a conjugate gradient solver. We have found the standard domain-wall action is the least effective. The Zolotarev versions of Con- 
tinued Fraction (with $H=H_{w}$ and $H_{T}$ ) appear to be among the best. Future work will focus on dynamical fermion implementations of this operator.

\section{Acknowledgments}

We gratefully acknowledge support for this work from DOE contract DE-FC02-94ER40818 and DE-AC05-84ER40150 under which the Southeastern Universities Research Association operates the Thomas Jefferson National Accelerator Facility (RGE), from PPARC under grant number PPA/G/O/2002/00465 (BJ) and from the European Commission's Research Infrastructures activity of the Structuring the European Research Area programme, contract number RII3-CT-2003-506079 (HPC-Europa) (UW). We gratefully acknowledge valuable discussions with Richard Brower.

\section{References}

[1] D.B. Kaplan, Phys. Lett. B288, 342 (1992).

[2] Y. Shamir, Nucl. Phys. B406, 90 (1993).

[3] R. Narayanan and H. Neuberger, Nucl. Phys. B443, 305 (1995).

[4] H. Neuberger, Phys. Rev. D57:5417-5433, 1998.

[5] H. Neuberger, Phys. Rev. Lett. 81:4060-4062, 1998.

[6] R.G. Edwards, U.M. Heller, R. Narayanan, Nucl. Phys. B540 (1999) 457.

[7] A. Borici, Proc. 3rd QCDNA Workshop, Springer, Berlin, 2005.

[8] H. Neuberger, Phys. Rev. D60:065006, 1999, hep-lat/9901003.

[9] A. Borici, et. al., Nucl. Phys. B (Proc. Suppl.) 106:757-759, 2002.

[10] U. Wenger, Proc. 3rd QCDNA Workshop, Springer, Berlin, 2005.

[11] A. Borici, Nucl. Phys. B (Proc. Suppl.) 83 (2000) 771-773, and hep-lat/9912040.

[12] R. Edwards and U. Heller, Phys. Rev. D63:094505, 2001.

[13] T.-W. Chiu, Phys. Rev. Lett.90:071601, 2003.

[14] R.C. Brower, H. Neff, K. Orginos, Nucl. Phys. B (Proc. Suppl.) 140 (2005) 686-688.

[15] P. Pandey, C. Kenney, A.J. Laub, Int. J. of High Speed Computing 2 (1990) 181.

[16] S. Dürr, Ch. Hoelbling, U. Wenger, hep-lat/0506027.

[17] H. Neuberger and R. Narayanan, Phys. Rev. D62:074504, 2000.

[18] Y. Kikukawa and Noguchi, hep-lat/9902022.

[19] Y. Aoki, et.al., hep-lat/0411006, http://qcd.nersc.gov/configs/MILC/BNL/dir.html . 